\title{
Numerical Study of Particle Migration in Tube and Plane Poiseuille Flows
}

\author{
B.H. Yang ${ }^{1}$, J. Wang ${ }^{1}$, D.D. Joseph ${ }^{1}$, H.H. Hu ${ }^{2}$, T-W. Pan ${ }^{3}$ and R. \\ Glowinski $^{3}$ \\ ${ }^{1}$ Department of Aerospace Engineering and Mechanics, University of \\ Minnesota, Minneapolis, MN, 55455, USA \\ ${ }^{2}$ Department of Mechanical Engineering and Applied Mechanics, \\ University of Pennsylvania, Philadelphia, PA, 19104, USA \\ ${ }^{3}$ Department of Mathematics, University of Houston, Houston, TX 77204, \\ USA
}

\begin{abstract}
The lateral migration of a single spherical particle in tube Poiseuille flow is simulated by ALE scheme, along with the study of the movement of a circular particle in plane Poiseuille flow with consistent dimensionless parameters. These particles are rigid and neutrally buoyant. A lift law $L=C U_{s}\left(\Omega_{s}-\Omega_{s e}\right)$ analogous to $L=\rho U \Gamma$ is validated in both two dimensions and three dimensions here. A method of constrained simulation is used to generate data which is processed for correlation formulas for the lift force, slip velocity, and equilibrium position. Our formulas predict the change of sign of the lift force which is necessary in the Segrè-Silberberg effect. Correlation formulas are compared between tube and plane Poiseuille flows by fixing the dimensionless size of particle and the Reynolds number. Our work provides a valuable reference for a better understanding of the migration of particle in Poiseuille flows and the Segrè-Silberberg effect.
\end{abstract}

\section{Introduction}

The literature on the migration of rigid particles in shear flow has been reviewed by Yang, Wang, Joseph, Hu, Pan and Glowinski (2005) and else where and will not be reviewed here. Yang et al. (2005) used the ALE scheme 
to study the lift force on a neutrally buoyant sphere in tube Poiseuille flow. They validated the lift law in three dimensions and established a general procedure for obtaining correlation formulas from numerical experiments. Their correlation formulas and predictions obtained good agreement with the literature.

The main goal of this work is to correlate the lift laws in two dimensions and three dimensions simultaneously by fixing some important dimensionless parameters such as Reynolds number and the dimensionless size of particle. Another goal is to study the analog and the difference between the migration of a spherical particle in tube Poiseuille flow and that of a circular particle in plane Poiseuille flow by analyzing the results obtained from the same procedure of data interrogation.

\section{Governing equations and dimensionless parameters}

(a)

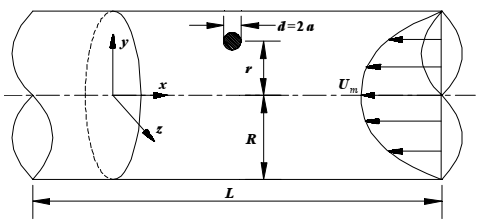

(b)

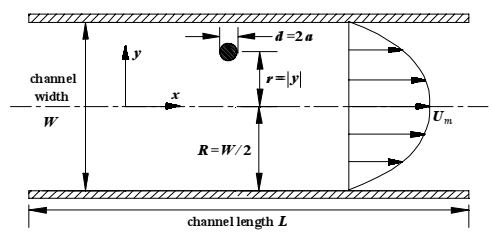

Fig. 1. Sketches for the problem of (a) a rigid spherical particle in tube Poiseuille flow and (b) a rigid circular particle in plane Poiseuille flow.

The fluid-particle system is governed by the Navier-Stokes equations for the fluid and Newton's equations for rigid body motions. The dimensionless governing equations in a general three dimensional case are

$$
\begin{gathered}
R_{e}\left(\frac{\partial \mathbf{u}}{\partial \mathrm{t}}+(\mathbf{u} \cdot \nabla) \mathbf{u}\right)=-\nabla p+\nabla^{2} \mathbf{u}, \\
\frac{\rho_{\mathrm{p}}}{\rho_{\mathrm{f}}} R_{e} \frac{\mathrm{d} \mathbf{U}_{\mathrm{p}}}{\mathrm{dt}}=G \mathbf{e}_{\mathrm{g}}+\frac{6}{\pi} \int[-p \mathbf{1}+\boldsymbol{\tau}] \cdot \mathbf{n} \mathrm{d} \Gamma, \\
\frac{\rho_{\mathrm{p}}}{\rho_{\mathrm{f}}} R_{e} \frac{\mathrm{d} \boldsymbol{\Omega}_{\mathrm{p}}}{\mathrm{dt}}=\frac{60}{\pi} \int\left(\mathbf{x}-\mathbf{X}_{\mathrm{p}}\right) \times([-p \mathbf{1}+\boldsymbol{\tau}] \cdot \mathbf{n}) \mathrm{d} \Gamma .
\end{gathered}
$$

The dimensionless parameters are

$$
\begin{gathered}
R_{e}=\frac{\rho_{f} V(2 a)}{\mu}=\frac{\rho_{f} \dot{\gamma}_{w}(2 a)^{2}}{\mu}=\frac{8 a^{2} \rho_{f} U_{m}}{\mu R}, \text { the Reynolds number; } \\
G=\frac{\left(\rho_{p}-\rho_{f}\right) g(2 a)^{2}}{\mu V}, \text { the gravity number; }
\end{gathered}
$$




$$
\rho_{p} / \rho_{f}, \text { the density ratio. }
$$

It is convenient to carry out the analysis of correlations in terms of dimensionless forms of correlating parameters. The ratio of the particle radius $a$ to tube radius $R$ and the dimensionless radial position $\bar{r}$ are defined by

$$
\bar{a}=a / R, \bar{r}=r / R \text {. }
$$

The dimensionless lift is given by

$$
\bar{L}=\frac{6 \rho_{f}}{\pi \mu^{2}} L \cdot
$$

Relative motions between the fluid and the particle, which may be characterized by slip velocities, are essential to understand the lift force on the particle. We use $U_{p}$ and $\Omega_{p}$ to denote the translational and angular velocities of the particle at steady state. The slip velocities are defined as:

$$
\begin{gathered}
U_{s}=U_{f}-U_{p}, \text { the slip velocity; } \\
\Omega_{s}=\Omega_{p}-\Omega_{f}=\Omega_{p}+\dot{\gamma} / 2, \text { the slip angular velocity, }
\end{gathered}
$$

where $U_{f}$ and $\dot{\gamma}$ are the fluid velocity and the local shear rate evaluated at the location of the particle center in the undisturbed flow.

We express the flow quantities $U_{m}, U_{s}, \Omega_{s}, \Omega_{s e}$ in the form of Reynolds numbers. A flow Reynolds number is given by

$$
\bar{U}_{m}=\frac{\rho_{f} U_{m} R}{\mu}=\frac{R_{e}}{8 \bar{a}^{2}} .
$$

Slip velocity Reynolds numbers are defined as

$$
\bar{U}_{s}=\rho_{f} U_{s}(2 a) / \mu, \bar{\Omega}_{s}=\rho_{f} \Omega_{s}(2 a)^{2} / \mu, \bar{\Omega}_{s e}=\rho_{f} \Omega_{s e}(2 a)^{2} / \mu .
$$

A dimensionless form of the product $U_{s}\left(\Omega_{s}-\Omega_{s e}\right)$ which enters into our lift law is given as the product $\bar{U}_{s}\left(\bar{\Omega}_{s}-\bar{\Omega}_{s e}\right)$.

We call the reader's attention to the fact that the flow is in the negative $x$ direction in our three dimensional simulation (see figure 1(a)). The symbol $U_{m}$ in (3) and (10) should be understood as the magnitude of the fluid velocity at the tube centerline. Similarly, we use the magnitude of $U_{f}$ and $U_{p}$ to calculate the slip velocity $U_{s}$ defined in (8). We shall focus on the steady state flow of a neutrally buoyant spherical particle, in which the left side of (2) and the term $G \mathbf{e}_{g}$ in (2) vanish. Thus, $R_{e}$ and $\bar{a}$ are the two parameters at play.

Here, we do not describe again the equations and parameters in two dimensions. Interested readers are referred to Wang and Joseph (2003) or Joseph and Ocando (2002) or Patankar, Huang, Ko and Joseph (2001) for details. The only change is that the coordinate is at the centerline of channel in this paper.

In the plane Poiseuille flow, the Reynolds number is 


$$
R_{e}=\frac{\rho_{f} V(2 a)}{\mu}=\frac{2 \rho_{f} \dot{\gamma}_{w}(2 a)^{2}}{\mu}=\frac{\rho_{f} W(2 a)^{2} \bar{p}}{\mu^{2}},
$$

where $\bar{p}$ is the constant pressure gradient. We also introduce the dimensionless parameter $\bar{r}$ to the two dimensional cases,

$$
\bar{r}=\frac{|y|}{R}=\frac{|y|}{W / 2},
$$

where $W$ is the width of channel and $R$ is half of the channel width. The dimensionless lift is given by

$$
\bar{L}=\frac{6 \rho_{f}(2 a)}{\pi \mu^{2}} L
$$

Other dimensionless quantities, such as $\bar{U}_{s}, \bar{\Omega}_{s}$ and $\bar{\Omega}_{s e}$, are in the same expressions to those in the three dimensions.

\section{Correlations from the numerical simulation}

Numerical experiments using constrained simulation provide us with the distribution of the lift force and particle velocity in the tube and the position and velocity of the particle at equilibrium. We develop correlations for these quantities in this section. The key correlation is for the lift force, which shows the dependence of the lift force on the slip angular velocity discrepancy $\Omega_{s}-\Omega_{s e}$. The lift force correlation predicts the change of sign of the lift force, which is necessary to explain the two-way migration in the Segrè-Silberberg effect. The correlations for the equilibrium state of the particle are also of interest, because they may be used to predict the position and the velocities of the particle at equilibrium.

\section{Correlation for the lift force}

The steady state values of the lift forces on a particle at different radial positions computed in constrained simulation are plotted in figure 2 for a spherical particle with the radius ratio $\bar{a}=0.15$ in three-dimensional tube Poiseuille flow. The same correlations for the migration of a circular particle in two-dimensional plane Poiseuille flow are also given in this figure. The positive direction of the lift force is in the negative $\mathbf{e}_{r}$ direction. In other words, $\bar{L}$ is positive when pointing to the centerline and negative when pointing away from the centerline. 
(a)

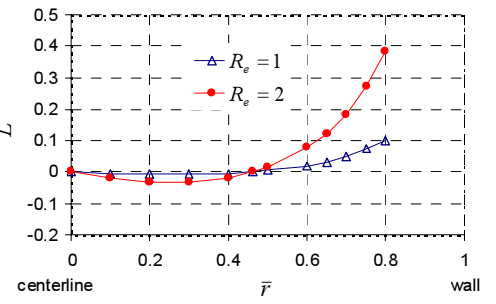

(c)

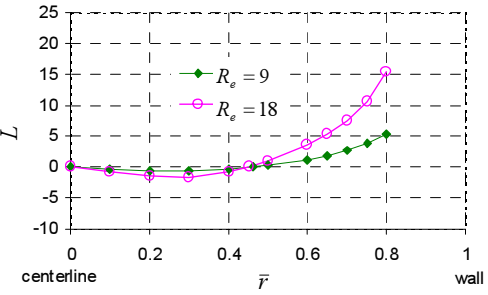

(e)

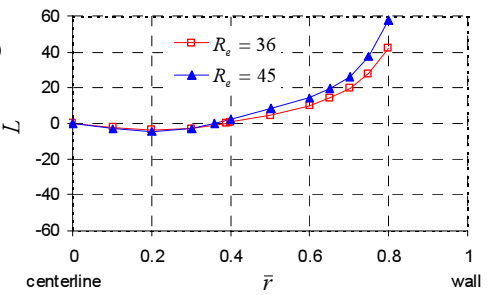

(b)

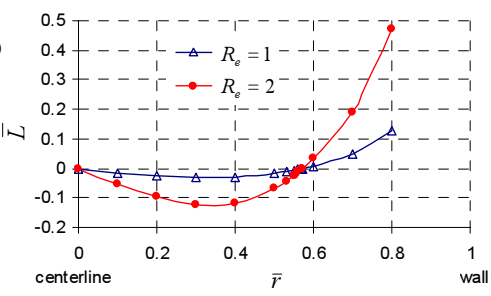

(d)

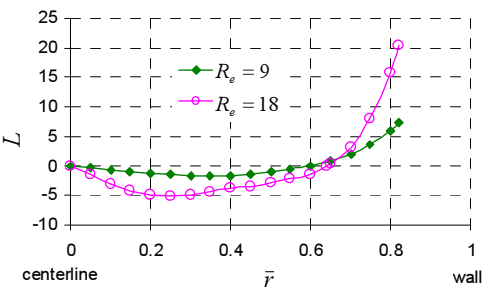

(f)

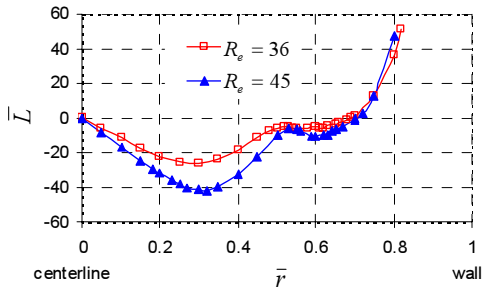

Fig. 2. The dimensionless lift force $\bar{L}$ at different radial positions for a particle with the radius ratio $\bar{a}=\mathbf{0 . 1 5}$. The curves in (a), (c) and (e) are for the migration of a circular particle in plane Poiseuille flow with different Reynolds numbers; the curves in (b), (d) and (f) are for the migration of a spherical particle in tube Poiseuille flow.

The equilibrium positions of a neutrally buoyant particle are the points where $\bar{L}=0$. The stability of the equilibrium at a zero-lift point can be determined from the slope of the $\bar{L}$ vs. $\bar{r}$ curve. The centerline is on a negative-slope branch of the $\bar{L}$ vs. $\bar{r}$ curve. When a particle is disturbed away from the centerline, the lift force is negative and drives the particle further away from the centerline. Therefore the centerline is an unstable equilibrium position. The other zero-lift point is between the centerline and the wall and it is on a positive-slope branch of the curve. When the particle is disturbed away from this point, the lift force tends to push the particle back. Thus the zero-lift point between the centerline and the wall is a stable equilibrium position. It is a surprise to see that the stable equilibrium position $\bar{r}_{e}$ moves towards the wall 
as the Reynolds numbers increases for the three-dimensional cases but away from the wall for the two-dimensional cases.

We discuss the three-dimensional cases with the radius ratio $\bar{a}=0.15$. When the Reynolds number is small $\left(R_{e}=1,2,9\right.$ or 18$)$, only one stable branch and one unstable branch can be observed in the $\bar{L}$ vs. $\bar{r}$ curves (figure 2(b), 2(d)). For higher Reynolds numbers, the distributions of the lift force as a function of the radial position become more complicated (figure 2(f)). A refined mesh was necessary to obtain converged results at high $R_{e}$.

We seek expressions for the lift force in terms of the slip velocities. The slip velocity Reynolds numbers have been defined in (11). We plot $\bar{\Omega}_{s}-\bar{\Omega}_{s e}$ at different radial positions in figure 3 for a particle with $\bar{a}=0.15$ for both the two-dimensional and the three-dimensional cases. Comparison of figure 2 and figure 3 shows that the quantity $\bar{\Omega}_{s}-\bar{\Omega}_{s e}$ always changes sign above and below the equilibrium position for either the two dimensions or the three dimensions.
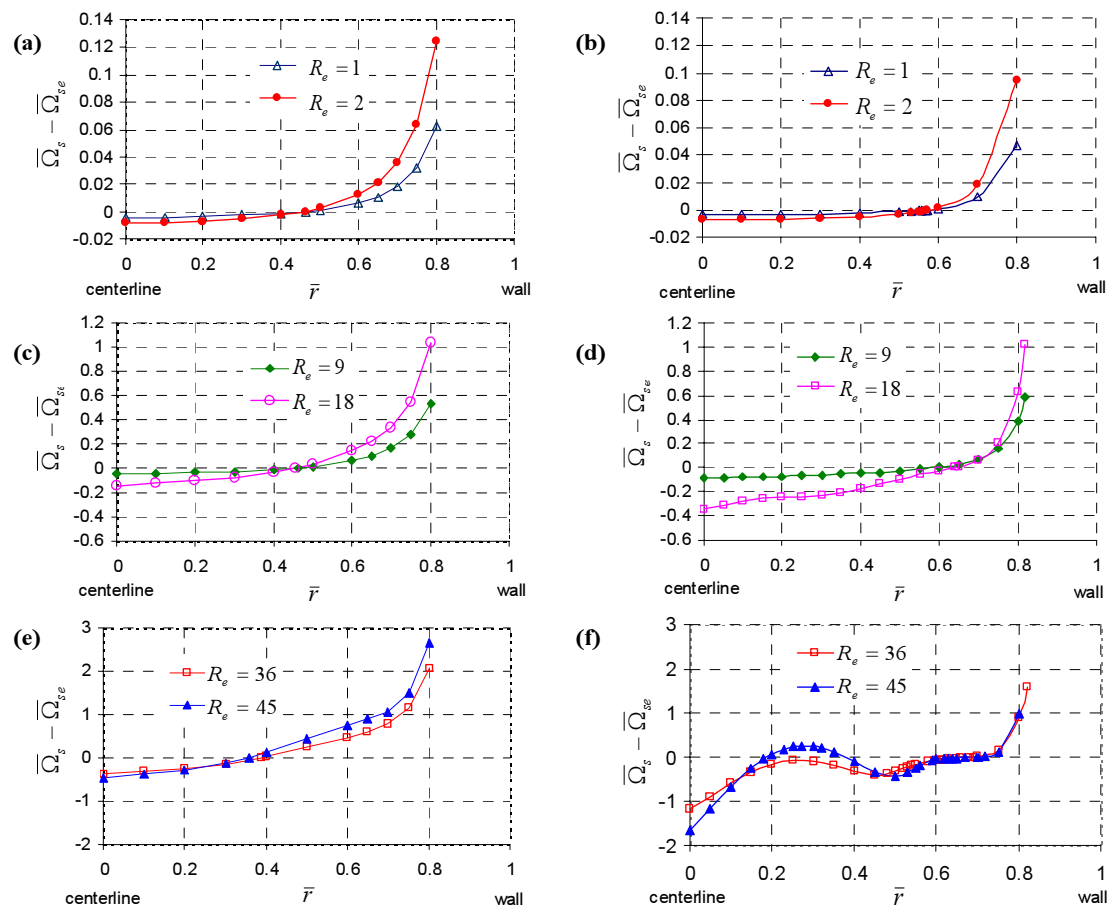

(f)

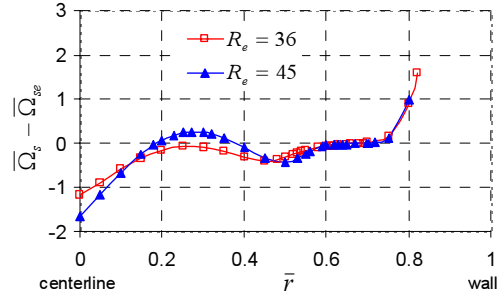


Fig. 3. The dimensionless slip angular velocity discrepancy at different radial positions for a particle with $\bar{a}=\mathbf{0 . 1 5}$. The curves in (a), (c) and (e) are for the migration of a circular particle in plane Poiseuille flow with different Reynolds numbers; the curves in (b), (d) and (f) are for the migration of a spherical particle in tube Poiseuille flow.

The lift $\bar{L}$ changes sign with the discrepancy $\bar{\Omega}_{s}-\bar{\Omega}_{s e}$ near the stable equilibrium position at all the Reynolds numbers. The lift correlation is developed in the region near the stable equilibrium position.

We seek correlations between the lift force $\bar{L}$ and the product

$$
F=\bar{U}_{s}\left(\bar{\Omega}_{s}-\bar{\Omega}_{s e}\right) \text {. }
$$

From our data, we noted that in the vicinity of the stable equilibrium position, the relation between $\bar{L}$ and $F$ may be represented by a linear correlation:

$$
\bar{L}\left(\bar{r}, R_{e}, \bar{a}\right)=k\left(R_{e}, \bar{a}\right) F\left(\bar{r}, R_{e}, \bar{a}\right),
$$

where $k$ is the proportionality coefficient which depends on the Reynolds number and the radius ratio $\bar{a}$. Some examples of the linear correlation between $\bar{L}$ and $F$ are plotted in figure 4 . The linear correlation (16) is not valid far away from the equilibrium position.

(a)

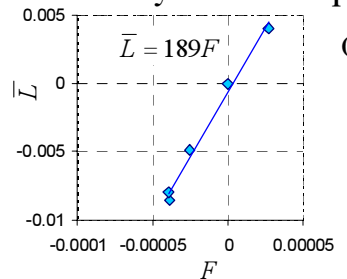

(d)

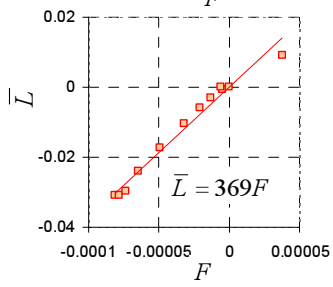

(b)

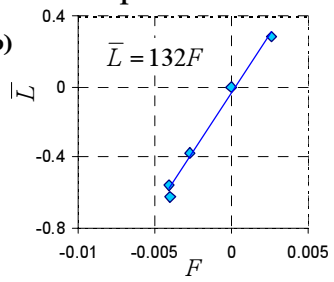

(e)

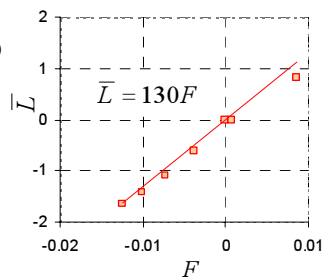

(c)

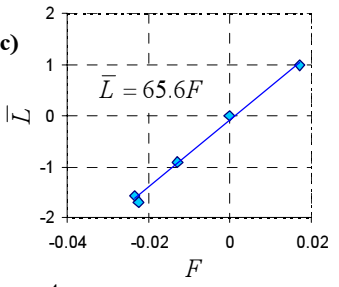

(f)

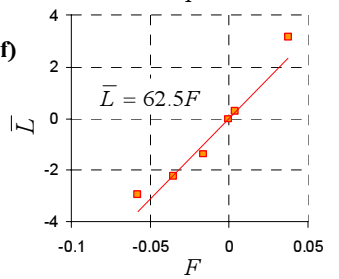

Fig. 4. The linear correlation between $\bar{L}$ and $F$ in the vicinity of the stable equilibrium position of a neutrally buoyant particle. (a) $R_{e}=1, \bar{a}=\mathbf{0 . 1 5}$, plane Poiseuille flow; (b) $R_{e}=9, \bar{a}=0.15$, plane Poiseuille flow; (c) $R_{e}=18, \bar{a}=0.15$, plane Poiseuille flow; (d) $R_{e}=1, \bar{a}=0.15$, tube Poiseuille flow; (e) $R_{e}=9, \bar{a}=$ 0.15, tube Poiseuille flow; (f) $R_{e}=18, \bar{a}=0.15$, tube Poiseuille flow.

We use power laws to fit the expressions for $k$ in terms of the Reynolds number and then obtain the linear correlations between $\bar{L}$ and $F$ by equation 
(16). To reveal the dependence of the lift force on the slip velocities explicitly, we substitute the definitions of $\bar{L}$ and $F$ into these correlations and then obtain the lift laws in table 2.

\begin{tabular}{|c|c|c|}
\hline \multicolumn{3}{|c|}{ Circular particle migrates in plane Poiseuille flow } \\
\hline range of $R_{e}$ & $\bar{a}=0.1$ & $\bar{a}=0.15$ \\
\hline$k=\bar{L} / F$ & $1 \leq R_{e} \leq 18$ & $1 \leq R_{e} \leq 45$ \\
\hline$L=\frac{\pi}{4} \frac{\mu^{2}}{\rho_{f}(2 a)} \bar{L}$ & $L=419 R_{e}^{-0.757}$ & $k=272 R_{e}^{-0.609}$ \\
\hline \multicolumn{2}{|c|}{ Spherical particle migrates in tube Poiseuille flow } \\
\hline \multicolumn{2}{|c|}{$\bar{a}=0.1$} & $\bar{a}=0.15$ \\
\hline range of $R_{e}$ & $1 \leq R_{e} \leq 12$ & $1 \leq R_{e} \leq 22.5$ \\
\hline$k=\bar{L} / F$ & $k=809 R_{e}^{-0.604} \rho_{f} U_{s}\left(\Omega_{s}-\Omega_{s e}\right)(2 a)^{2}$ & $L=214 R_{e}^{-0.609} \rho_{f} U_{s}\left(\Omega_{s}-\Omega_{s e}\right)(2 a)^{2}$ \\
\hline$L=\frac{\pi}{6} \frac{\mu^{2}}{\rho_{f}} \bar{L}$ & $L=424 R_{e}^{-0.604} \rho_{f} U_{s}\left(\Omega_{s}-\Omega_{s e}\right)(2 a)^{3}$ & $L=236 R_{e}^{-0.658} \rho_{f} U_{s}\left(\Omega_{s}-\Omega_{s e}\right)(2 a)^{3}$ \\
\hline
\end{tabular}

Table 1. Lift laws for the migration of a single neutrally-buoyant particle with $\bar{a}=$ 0.1 and $\bar{a}=0.15$ in plane and tube Poiseuille flows.

The lift force in our correlation is on a freely rotating particle translating at steady velocity. Thus correlations in table 1 apply to particles with zero acceleration. For a migrating particle with substantial acceleration, these correlations may not be valid.

\section{Correlations for slip velocity $U_{s}$ and slip angular velocity $\Omega_{\mathrm{s}}$}

Besides the lift force on the particle, the translational and the angular velocities of the particle at steady state are also of interest. We use power laws to fit the correlations between the slip velocities and the Reynolds number. All of coefficients in the power law correlations can be explicitly expressed in terms of $\bar{r}$. Details about the construction of the correlations for the slip velocity $U_{s}$ and the slip angular velocity $\Omega_{s}$ can be found in Yang et al. (2005) and will not be shown here. The final correlations for $U_{s}$ and $\Omega_{s}$ and the corresponding applicable ranges are listed in table 2. 


\begin{tabular}{|c|c|c|}
\hline \multicolumn{3}{|c|}{ Circular particle migrates in plane Poiseuille flow } \\
\hline & $\bar{a}=0.1$ & $\bar{a}=0.15$ \\
\hline range of $\bar{r}$ & $0.10 \leq \bar{r} \leq 0.85$ & $0.10 \leq \bar{r} \leq 0.80$ \\
\hline $\bar{U}_{s}$ & $\bar{U}_{s}=4.3 \times 10^{-3} \exp (3.2 \bar{r}) R_{e}^{-0.45 \bar{r}+1.1}$ & $\bar{U}_{s}=6.8 \times 10^{-3} \exp (3.0 \bar{r}) R_{e}^{-0.27 \bar{r}+1.1}$ \\
\hline $\bar{\Omega}_{s}$ & $\bar{\Omega}_{s}=6.4 \times 10^{-5} \exp (7.3 \bar{r}) R_{e}^{-0.95 \bar{r}+1.9}$ & $\bar{\Omega}_{s}=1.3 \times 10^{-4} \exp (7.3 \bar{r}) R_{e}^{-0.69 \bar{r}+1.6}$ \\
\hline \multicolumn{3}{|c|}{ Spherical particle migrates in tube Poiseuille flow } \\
\hline \multicolumn{2}{|c|}{$\bar{a}=0.1$} & $\bar{a}=0.15$ \\
\hline range of $\bar{r}$ & $0.05 \leq \bar{r} \leq 0.85$ & $0.10 \leq \bar{r} \leq 0.80$ \\
\hline $\bar{U}_{s}$ & $\bar{U}_{s}=7.4 \times 10^{-3} \exp (2.1 \bar{r}) R_{e}^{-1.4 \bar{r}+1.9}$ & $\bar{U}_{s}=1.1 \times 10^{-2} \exp (2.2 \bar{r}) R_{e}^{-1.1 \bar{r}+1.7}$ \\
\hline $\bar{\Omega}_{s}$ & $\bar{\Omega}_{s}=6.8 \times 10^{-6} \exp (9.6 \bar{r}) R_{e}^{-3.3 \bar{r}+3.9}$ & $\bar{\Omega}_{s}=2.1 \times 10^{-5} \exp (9.2 \bar{r}) R_{e}^{-2.1 \bar{r}+2.8}$ \\
\hline
\end{tabular}

Table 2. Correlations of slip velocity and slip angular velocity for the migration of a single neutrally-buoyant particle with $\bar{a}=0.1$ and $\bar{a}=0.15$ in plane Poiseuille flow and tube Poiseuille flow.

\section{Correlations for parameters at equilibrium}

The equilibrium state of a particle is always the focus of the study of particle migration. We obtain the particle parameters at stable equilibrium, such as the equilibrium position $\bar{r}_{e}$, the slip velocity $U_{s e}$ and the slip angular velocity $\Omega_{s e}$ by unconstrained simulation and find that they may be correlated to the Reynolds number. We summarize the particle parameters at stable equilibrium in table 3 .

\begin{tabular}{|c|c|c|c|c|c|c|c|c|c|}
\hline \multirow[t]{2}{*}{$\bar{a}$} & \multirow[t]{2}{*}{$R_{e}$} & \multicolumn{4}{|c|}{$\begin{array}{c}\text { Circular particle migrates in plane } \\
\text { Poiseuille flow }\end{array}$} & \multicolumn{4}{|c|}{$\begin{array}{l}\text { Spherical particle migrates in tube } \\
\text { Poiseuille flow }\end{array}$} \\
\hline & & $\bar{U}_{m}$ & $\overline{\bar{r}_{e}}$ & $\bar{\Omega}_{s e}$ & $\bar{U}_{s e}$ & ${\overline{U_{m}}}$ & $\overline{r_{e}}$ & $\bar{\Omega}_{s e}$ & $\bar{U}_{s e}$ \\
\hline 0.05 & 2 & - & - & - & - & 100 & 0.731 & 0.00710 & 0.0247 \\
\hline \multirow{10}{*}{0.1} & 1 & 1.25 & 0.478 & 0.00210 & 0.0155 & 12.5 & 0.603 & 0.00188 & 0.0219 \\
\hline & 2 & 2.5 & 0.478 & 0.00460 & 0.0317 & 25 & 0.608 & 0.00509 & 0.0444 \\
\hline & 4 & 5 & 0.476 & 0.0124 & 0.0661 & 50 & 0.638 & 0.0209 & 0.0901 \\
\hline & 6 & - & - & - & - & 75 & 0.661 & 0.0498 & 0.152 \\
\hline & 8 & 10 & 0.456 & 0.0406 & 0.134 & 100 & 0.674 & 0.0901 & 0.470 \\
\hline & 10 & - & - & - & - & 125 & 0.684 & 0.139 & 0.712 \\
\hline & 12 & 15 & 0.413 & 0.0724 & 0.194 & 150 & 0.708 & 0.202 & 0.296 \\
\hline & 18 & 22.5 & 0.357 & 0.110 & 0.276 & - & - & - & - \\
\hline & 80 & 100 & 0.222 & 0.499 & 1.01 & - & - & - & - \\
\hline & 180 & 225 & 0.174 & 1.09 & 2.12 & - & - & - & - \\
\hline
\end{tabular}




\begin{tabular}{|c|c|c|c|c|c|c|c|c|c|}
\hline \multirow{11}{*}{0.15} & 1 & 0.556 & 0.463 & 0.00419 & 0.0225 & 5.56 & 0.573 & 0.00354 & 0.0338 \\
\hline & 2 & 1.11 & 0.463 & 0.00849 & 0.0452 & 11.1 & 0.573 & 0.00765 & 0.0675 \\
\hline & 9 & 5 & 0.464 & 0.0491 & 0.214 & 50 & 0.601 & 0.0861 & 0.306 \\
\hline & 13.5 & - & - & - & - & 75 & 0.623 & 0.197 & 0.482 \\
\hline & 18 & 10 & 0.454 & 0.145 & 0.439 & 100 & 0.642 & 0.342 & 0.730 \\
\hline & 22.5 & - & - & - & - & 125 & 0.657 & 0.513 & 0.785 \\
\hline & 27 & - & - & - & - & 150 & 0.670 & 0.705 & 1.07 \\
\hline & 36 & 20 & 0.388 & 0.368 & 0.799 & 200 & 0.691 & 1.16 & 1.18 \\
\hline & 45 & 25 & 0.359 & 0.455 & 0.967 & 250 & 0.700 & 1.67 & 1.74 \\
\hline & 180 & 100 & 0.234 & 1.70 & 3.14 & - & - & - & - \\
\hline & 360 & 200 & 0.190 & 3.21 & 5.62 & - & - & - & - \\
\hline 0.2 & 32 & - & - & - & - & 100 & 0.598 & 0.793 & 1.74 \\
\hline 0.25 & 50 & - & - & - & - & 100 & 0.567 & 1.49 & 2.84 \\
\hline
\end{tabular}

Table 3. Particle parameters at stable equilibrium: the equilibrium position $\bar{r}_{e}$, the dimensionless slip angular velocity $\bar{\Omega}_{s e}=\rho_{f} \Omega_{s e}(2 a)^{2} / \mu$ and the dimensionless slip velocity $\bar{U}_{s e}=\rho_{f} U_{s e}(2 a) / \mu$.

The correlations for the equilibrium position $\bar{r}_{e}$ are shown in figure 5 . In two dimensions, multiple power law fittings are used in different ranges of Reynolds numbers (figure 5(b)). As mentioned before, $\bar{r}_{e}$ moves closer to the wall as the Reynolds number increases for the three dimensional cases but moves to the centerline for the two dimensional cases.
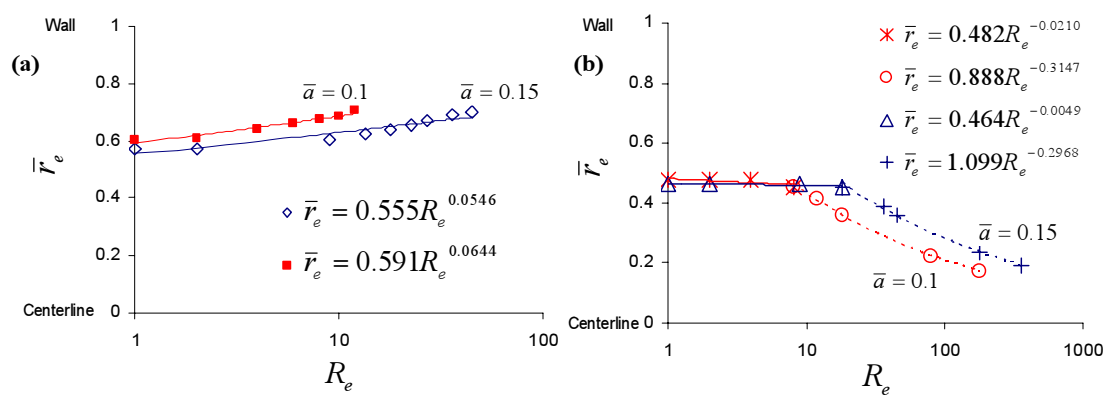

Fig. 5. The stable equilibrium position $\bar{r}_{e}$ of a neutrally buoyant particle as a function of the Reynolds number in (a) tube Poiseuille flow and (b) plane Poiseuille flow.

Figure 6 shows that power law correlations also exist between the dimensionless slip angular velocity at equilibrium $\bar{\Omega}_{\text {se }}$ and the Reynolds 
number $R_{e}$ for either the two dimensions or the three dimensions. These correlations are important because they give explicitly the slip angular velocity when the particle is at stable equilibrium.
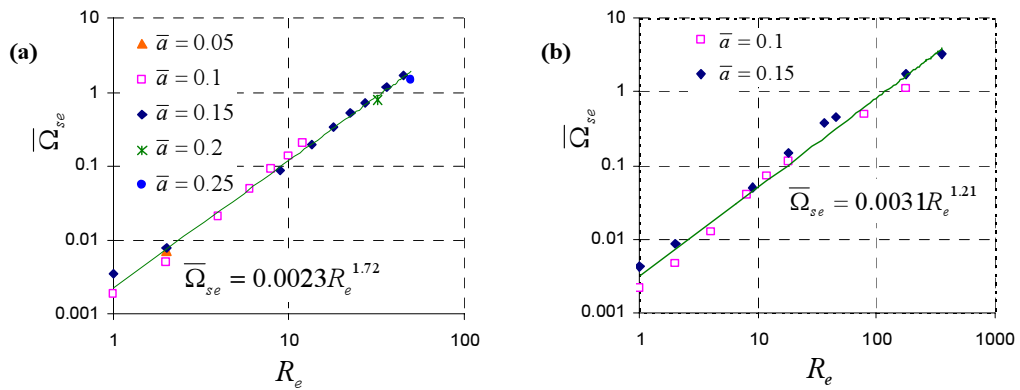

Fig. 6. The correlations between $\bar{\Omega}_{s e}$ and the Reynolds number $R_{e}$ for the migration of (a) a spherical particle in tube Poiseuille flow and (b) a circular particle in plane Poiseuille flow.

The correlations for parameters at equilibrium are summarized in table 4 .

\begin{tabular}{|c|c|c|}
\hline & $\begin{array}{c}\text { Circular particle migrates in plane } \\
\text { Poiseuille flow }\end{array}$ & $\begin{array}{l}\text { Spherical particle migrates in tube } \\
\text { Poiseuille flow }\end{array}$ \\
\hline $\bar{\Omega}_{s e}$ & $\begin{array}{c}\bar{\Omega}_{s e}=0.0031 R_{e}^{1.21} \\
\text { (for } 0.10 \leq \bar{a} \leq 0.15 \text { ) }\end{array}$ & $\begin{array}{c}\bar{\Omega}_{s e}=0.0023 R_{e}^{1.72} \\
\text { (for } 0.05 \leq \bar{a} \leq 0.25 \text { ) }\end{array}$ \\
\hline$\Omega_{s e}$ & $\begin{array}{c}\Omega_{s e}=0.0031 R_{e}^{1.21} \mu /\left(\rho_{f} 4 a^{2}\right) \\
\quad(\text { for } 0.10 \leq \bar{a} \leq 0.15)\end{array}$ & $\begin{aligned} \Omega_{s e} & =0.0023 R_{e}^{1.72} \mu /\left(\rho_{f} 4 a^{2}\right) \\
& (\text { for } 0.05 \leq \bar{a} \leq 0.25)\end{aligned}$ \\
\hline $\bar{r}_{e}$ & $\begin{array}{c}\bar{r}_{e}=0.482 R_{e}^{-0.0210} \\
\text { (for } \bar{a}=0.1 \text { and } 1 \leq R_{e} \leq 8 \text { ); } \\
\bar{r}_{e}=0.888 R_{e}^{-0.3147} \\
\text { (for } \bar{a}=0.1 \text { and } 8 \leq R_{e} \leq 180 \text { ); } \\
\bar{r}_{e}=0.464 R_{e}^{-0.0049} \\
\text { (for } \bar{a}=0.15 \text { and } 1 \leq R_{e} \leq 18 \text { ); } \\
\bar{r}_{e}=1.099 R_{e}^{-0.0869} \\
\text { (for } \bar{a}=0.15 \text { and } 18 \leq R_{e} \leq 360 \text { ). }\end{array}$ & $\begin{array}{c}\bar{r}_{e}=0.591 R_{e}^{0.0644} \\
\text { (for } \bar{a}=0.1 \text { and } 1 \leq R_{e} \leq 12 \text { ); } \\
\bar{r}_{e}=0.555 R_{e}^{0.0546} \\
\text { (for } \bar{a}=0.15 \text { and } 1 \leq R_{e} \leq 45 \text { ). }\end{array}$ \\
\hline
\end{tabular}


Table 4. Correlations of particle parameters at equilibrium for the migration of a single neutrally-buoyant particle with $\bar{a}=0.1$ and $\bar{a}=0.15$ in plane Poiseuille flow and tube Poiseuille flow.

\section{Conclusion}

- A lift law $L=C U_{s}\left(\Omega_{s}-\Omega_{\text {se }}\right)$ analogous to $L=\rho U \Gamma$ of the classical aerodynamics is valid in both two dimensions and three dimensions.

- Equilibrium may be identified at the Segré-Silberberg radius at which the lift vanishes (for a neutrally buoyant particle).

- The slip angular velocity discrepancy $\Omega_{s}-\Omega_{s e}$ is the circulation for the free particle and it is shown to change sign at the equilibrium position where the lift reaches zero on its stable branch. The behaviors of $L$ and $\Omega_{s}-\Omega_{\text {se }}$ are very similar between the two dimensions and the three dimensions at comparatively low Reynolds numbers.

- The equilibrium position (the Segré-Silberberg radius) moves towards the wall as $R_{e}$ increases at each fixed $\bar{a}$ for the migration of a spherical particle in tube Poiseuille flow but moves away from the wall for the migration of a circular particle in plane Poiseuille flow.

\section{References}

[1] Joseph, D.D., Ocando, D. Slip Velocity and Lift. J. Fluid Mech. 454, 263-286, 2002.

[2] Patankar, N.A., Huang, P.Y., Ko, T., Joseph, D.D. Lift-off of a single particle in Newtonian and viscoelastic fluids by direct numerical simulation. J. Fluid Mech. 438, 67-100, 2001.

[3] Segrè, G., Silberberg, A. Radial Poiseuille flow of suspensions. Nature 189, 209, 1961.

[4] Segrè, G., Silberberg, A. Behavior of macroscopic rigid spheres in Poiseuille flow: Part I. J. Fluid Mech. 14, 136-157, 1962.

[5] Wang, J., Joseph, D.D. Lift forces on a cylindrical particle in plane Poiseuille flow of shear thinning fluids. Physics Fluids 15, 2267-2278, 2003.

[6] Yang, B.H., Wang, J., Joseph, D.D., Hu, H.H., Pan, T-W., Glowinski, R. Migration of a sphere in tube flow. J. Fluid Mech. accepted, 2005. 